This item was submitted to Loughborough's Research Repository by the author.

Items in Figshare are protected by copyright, with all rights reserved, unless otherwise indicated.

\title{
A Monte Carlo platform for the optical modeling of pulse oximetry
}

PLEASE CITE THE PUBLISHED VERSION

http://dx.doi.org/10.1117/12.699084

PUBLISHER

(c) SPIE

VERSION

AM (Accepted Manuscript)

\section{PUBLISHER STATEMENT}

This work is made available according to the conditions of the Creative Commons Attribution-NonCommercialNoDerivatives 4.0 International (CC BY-NC-ND 4.0) licence. Full details of this licence are available at: https://creativecommons.org/licenses/by-nc-nd/4.0/

\section{LICENCE}

CC BY-NC-ND 4.0

\section{REPOSITORY RECORD}

Azorin-Peris, Vicente, Sijung Hu, and Peter R. Smith. 2019. "A Monte Carlo Platform for the Optical Modeling of Pulse Oximetry”. figshare. https://hdl.handle.net/2134/22217. 
Proceedings of SPIE -- Volume 6446

Biomedical Applications of Light Scattering, Adam Wax, Vadim Backman, Editors, 64460T (Feb. 15, 2007)

\title{
A Monte Carlo Platform for the Optical Modelling of Pulse Oximetry
}

\author{
Vicente Azorin Peris ${ }^{*}$, Sijung Hu and Peter R Smith \\ Department of Electronic and Electrical Engineering, Loughborough University, Ashby Road, \\ Loughborough, Leicestershire, LE11 3TU, UK
}

\begin{abstract}
We investigated a custom Monte Carlo (MC) platform in the generation of opto-physiological models of motion artefact and perfusion in pulse oximetry. With the growing availability and accuracy of tissue optical properties in literatures, MC simulation of light-tissue interaction is providing increasingly valuable information for optical bio-monitoring research. Motion-induced artefact and loss of signal quality during low perfusion are currently the primary limitations in pulse oximetry. While most attempts to circumvent these issues have focused on signal post-processing techniques, we propose the development of improved opto-physiological models to include the characterisation of motion artefact and low perfusion. In this stage of the research, a custom MC platform is being developed for its use in determining the effects of perfusion, haemodynamics and tissue-probe optical coupling on transillumination at different positions of the human finger. The results of MC simulations indicate a useful and predictable output from the platform.
\end{abstract}

Keywords: Pulse Oximetry, Monte Carlo simulation, Optical Modelling, motion artefact, photoplethysmography

\section{INTRODUCTION}

Photoplethysmography (PPG) is an optical biomonitoring technique that non-invasively measures arterial pulsations invivo. Its ease of use and convenience make it an attractive area of research in the biomedical and clinical community. Among its applications, pulse oximetry - the determination of arterial oxygen saturation — is the most widespread thanks to its ability to alert the clinician of the presence of hypoxemia in real-time. ${ }^{1}$

\subsection{Current Pulse Oximetry}

Pulse oximetry has become a standard of patient monitoring during anaesthesia, in recovery rooms and under intensive care. $^{2}$ However, oximeters have a number of factors that lead to inaccurate readings and limit their applicability. As such, these are practable areas of research when attempting to increase the reliability and applicability of the technology.

Commercial pulse oximeters convert the ratio of normalised pulsatile components into an equivalent arterial oxygen saturation by means of a look-up table which is determined by comparison of oximeter readings with invasive blood gas measurements. ${ }^{3}$ This calibration is generally applied with oxygen saturation values above $70 \%$ due to serious health risks encountered by test subjects for saturations below this point. The limitation encountered due to health risks at saturations below $70 \%$ make it necessary for oximeter calibration schemes to rely on simulations and in-vitro measurements if precise calibration curves at low-saturation are required. ${ }^{4,5}$ A common source of PPG signal corruption is the inadvertent measurement of voluntary or involuntary movement of the patient. ${ }^{6}$ Motion artifact is a complex issue due to its contribution to the PPG signal being several orders of magnitude larger than that of the arterial pulsations, and to the spectral and temporal dependence of signal and artefact. ${ }^{7}$ In cases of low perfusion such as critically ill patients, the low signal-to-noise ratio (SNR) encountered under a lack of a strong pulsatile PPG signal component leads to inaccurate readings. ${ }^{2}$ Adequate knowledge of a patient's oxygenation is especially useful under these circumstances.

From an engineering perspective, most solutions to the above issues consist of additional characterisation of signal components. ${ }^{6-9}$ The success of these solutions depends heavily on the validity of the assumptions used to correlate the signals. Also, the adverse effects of these issues are generally quantified according to the sensitivity and specificity of oximeters under the relevant scenarios, often overlooking the mechanisms leading to such inaccuracies.

*V.Azorin-Peris@lboro.ac.uk; phone+44 1509227059 
Proceedings of SPIE -- Volume 6446

Biomedical Applications of Light Scattering, Adam Wax, Vadim Backman, Editors, 64460T (Feb. 15, 2007)

\subsection{Tissue Optical Modeling}

The operation principal of current pulse oximeters is commonly described using the Beer-Lambert model, where the measuring site is treated as a blood-filled cuvette with no scattering effects and the light sources are assumed to be monochromatic. ${ }^{10}$ The highly scattering nature of human tissue clearly contradicts these assumptions, but the model illustrates the common simplification process inherent in the area.

Numerous models that predict photon transport in tissue have been developed, ${ }^{11}$ most of which are simplified forms of the radiation transfer theory (RTT). The latter is commonly accepted as a sufficiently strict mathematical description of continuous wave $(\mathrm{CW})$ light propagation in a scattering medium ${ }^{12}$ and relies on the absorption and scattering coefficients $\mu_{\mathrm{a}}$ and $\mu_{\mathrm{s}}$, and anisotropy factor $g$ for an optical description of the medium. The diffusion approximation of RTT, also known as the $P_{1}$ approximation, ${ }^{13}$ assumes isotropic scattering, therefore relying on $\mu_{\mathrm{a}}$ and transport scattering coefficient $\mu_{\mathrm{s}}$,', where $\mu_{\mathrm{s}}{ }^{\prime}=\mu_{\mathrm{s}}(1-\mathrm{g})$. Monte Carlo (MC) radiation transport techniques are based on the stochastic nature of radiation interactions, and in the context of tissue optics, they provide a numerical solution of the RTT equation and the $P_{1}$ approximation. As a finite element method, it is suited for complex geometries and is capable of achieving very high degrees of accuracy at the expense of heavy computational load.

The accuracy of optical propagation models ultimately depends on that of the tissue optical properties. In recent years, several substantial investigations have led to the determination of increasingly accurate optical properties of the constituents of PPG monitoring sites in a range of wavelengths. ${ }^{11-18}$

\subsection{Aims and Objectives of Study}

We propose the combination of optical properties and standardised models of human anatomy and physiology in MC simulations of light transport to deliver a characterisation of the mechanisms and consequent systematic reduction of the effects of issues with an adverse effect to pulse oximetry. A MC platform has been created to perform light transport simulations on complex tissue geometries. The current phase of the investigation aims to characterise the distribution of transmitted light around the finger at the standard wavelengths of pulse oximeters. This data will be used to provide optimum source and sensor positions and will be the first step towards the characterisation of probe motion artefact in standard transmittance mode pulse oximetry.

\section{MATERIALS AND METHODS}

The structure and operation of the platform developed for MC raytracing of complex tissue geometries are illustrated in this section. An accurate 3D anatomical model (Zygote Media Group, USA) of a male adult finger composed of bones, tendons, nerves, arteries, veins and outer skin was hired in this investigation (see Fig. 2). The platform can be sectioned into three phases, each of which rely on an available software package (Fig. 1). 3D Studio MAX v.9.0 (Autodesk, USA) was employed to make modifications to the base model prior to simulation. These included the creation of five-layer skin in accordance to the skin model described by Tuchin et al., ${ }^{18}$ the creation of subcutaneous fat as a filler between the skin layers and all other internals, as well as the generation of separate files for each of the objects in the model. MC raytracing was performed by OptiCAD 10.0 (Opticad Corporation, USA). Post-processing was performed in MATLAB 7.1 (Mathworks Co., USA) algorithms customised for the output data of OptiCAD.

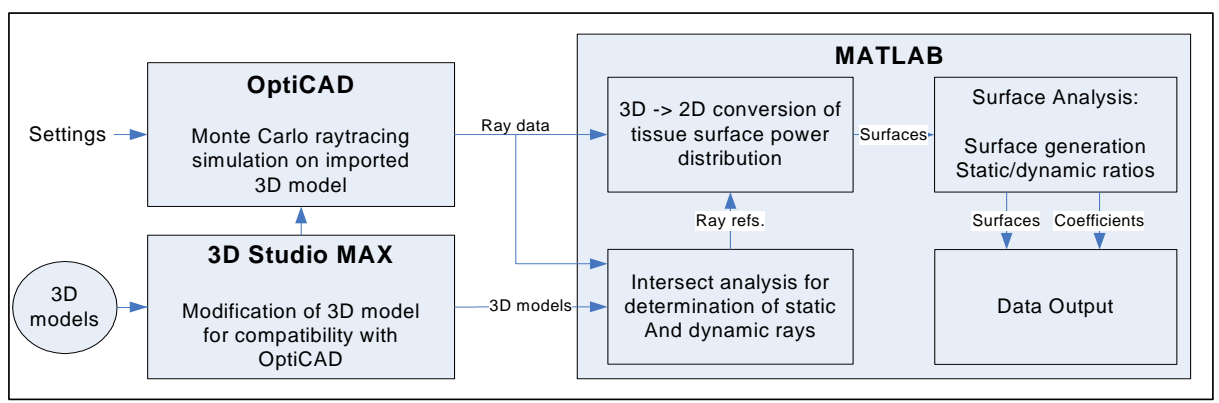

Figure 1. Block diagram of MC raytracing platform. 
Proceedings of SPIE -- Volume 6446

Biomedical Applications of Light Scattering, Adam Wax, Vadim Backman, Editors, 64460T (Feb. 15, 2007)

\subsection{Tissue Optical Properties for Monte Carlo}

OptiCAD treats the imported components of the 3D model as solids and allows the volumetric optical properties (absorption coefficient $\mu_{\mathrm{a}}$, scattering coefficient $\mu_{\mathrm{s}}$, and anisotropy factor $g$ ) of each to be defined. Transport scattering coefficients $\mu_{\mathrm{s}}$ ' at $633 \mathrm{~nm}$, derived from $\mu_{\mathrm{s}}{ }^{\prime}=\mu_{\mathrm{s}}(1-g)$, are used for all media in the model to significantly reduce raytracing time. While a range of sources contained optical properties of human tissues at $633 \mathrm{~nm}$, those at $850 \mathrm{~nm}$ were scarce in the literature. A series of ratios were taken from a range of sources which allowed the derivation of coefficients at $850 \mathrm{~nm}$ by means of scaling those at $633 \mathrm{~nm}$. This process aimed to ensure that the derived coefficients emulated the experimental conditions used in the determination of the readily available $633 \mathrm{~nm}$ coefficients. Only the surface optical properties of bone were considered, namely its diffuse reflectance and transmission. The absorption and scattering coefficients used in this simulation can be found in table I.

All raytraces were performed using a spherical light source with a lambertian intensity distribution. Reflection and refraction at interfaces resulting from refractive index mismatches are neglected. This ensures minimisation of inaccuracies due to the inherently difficult task of modeling the microscopic textures of interfaces, which pose a significant level of complexity in contrast to their minor contribution to the output. Sobol Sampling ${ }^{20}$ (Quasi-Monte Carlo Sampling) was used in order to increase the convergence rate of raytraces.

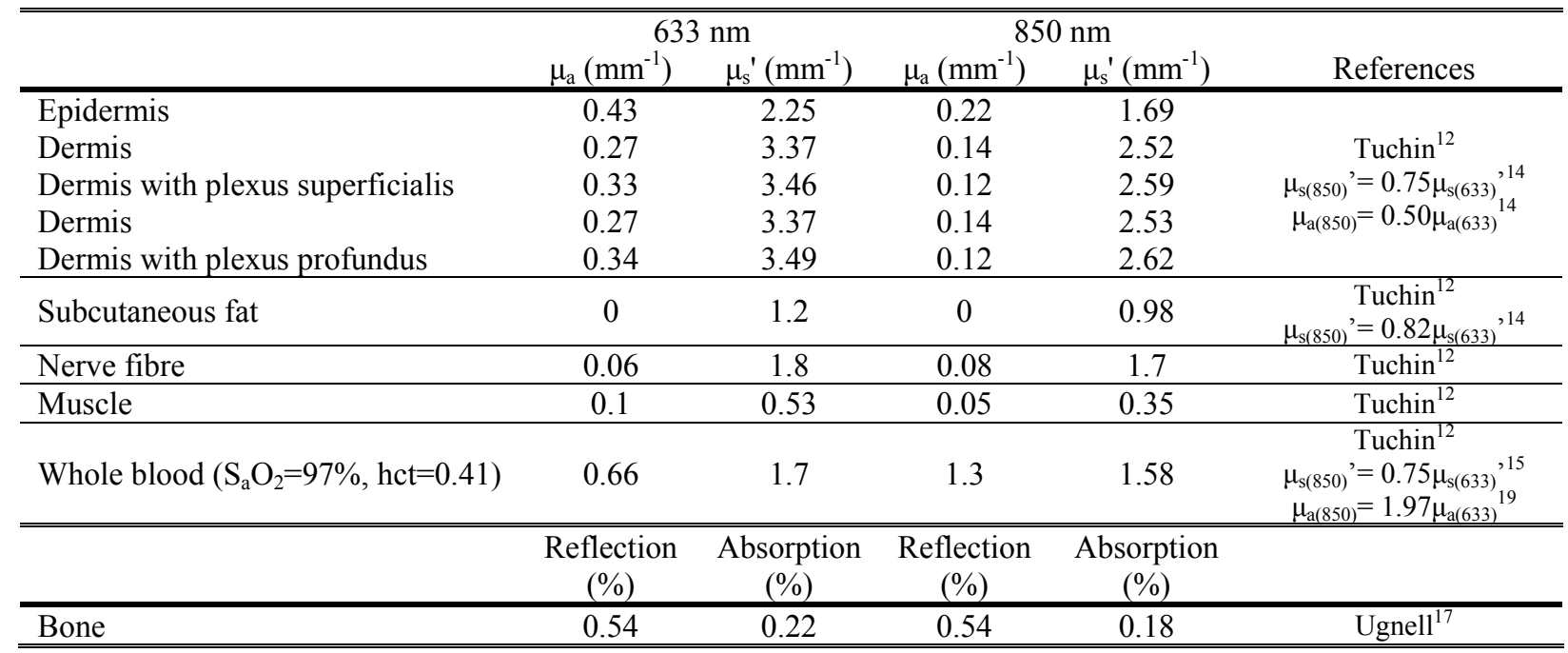

Table I: Optical properties used for elements of finger model in MC raytraces, sources of data, and ratios used to determine the coefficients at $850 \mathrm{~nm}$.

\subsection{Post-Processing}

Several algorithms were generated for the post-processing of output data from OptiCAD, all of which have been optimised in terms of speed, memory usage and large data handling capability. The core function is in charge of performing an intersect analysis on a specific component of the 3D model (e.g. arteries, plexus superficialis). This algorithm allows the labeling of rays whose intensity has been modulated by any medium in the model. For instance, in the case of artefact-free PPG, this information distinguishes localised light intensities that will vary due to the cardiovascular cycle from those that are static. The algorithm determines the changes in intensity $\Delta I_{\text {art }}$ of a ray due to absorption by a medium and stores the total change in intensity $\Sigma \Delta I_{\text {art }}$ due to the selected medium, as is the case when a ray traverses the medium several times. Determination of the exit points $(x, y, z)$ of all traced rays and their corresponding intensities $I_{\text {end }}$ is also performed. The following relationships are derived from this output data: When a ray does not traverse a time-varying medium, its intensity only contributes to the DC intensity at the measuring site, thus $I_{t(D C)}=I_{\text {end }}$. When a ray does traverse such a medium, its intensity contributes both to DC and AC intensities at the measuring site, where $I_{t(A C)}$ reaches its maximum and minimum values during diastole and systole respectively. To define $I_{t(A C)}$, it is assumed that arterial absorption at systole and diastole are both proportional to the total arterial absorption, resulting in two separate equations, $I_{t(A C)(s y s)}=\alpha \Sigma \Delta I_{a r t}$ and $I_{t(A C)(d i a)}=\beta \Sigma \Delta I_{\text {art }}$, where $\alpha$ and $\beta$ are the ratios of systolic and diastolic 
Proceedings of SPIE -- Volume 6446

Biomedical Applications of Light Scattering, Adam Wax, Vadim Backman, Editors, 64460T (Feb. 15, 2007)

absorption to total arterial absorption respectively. The DC intensity at the measuring site is corrected by defining it as $I_{t(D C)}=I_{\text {end }}-\left(I_{t(A C)(d i a)}-I_{t(A C)(\text { sys })}\right) / 2$.

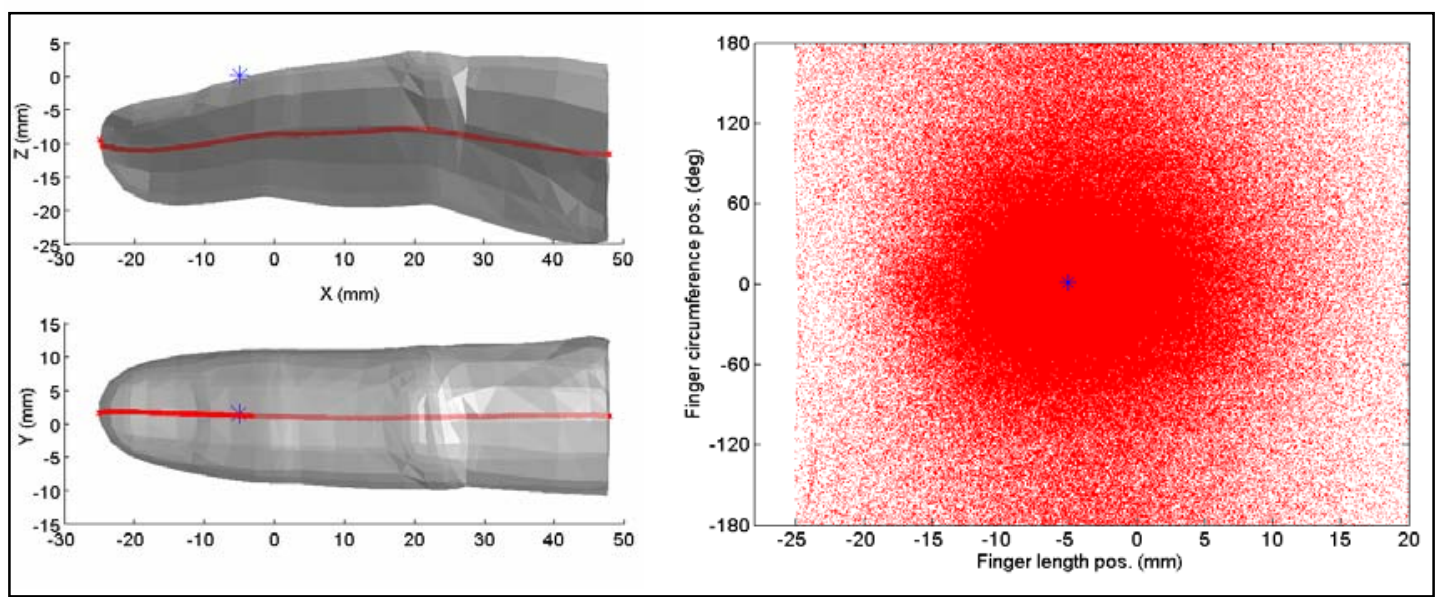

Figure 2. Outer skin model with central finger axis highlighted and resultant plot after conversion of transmitted ray coordinates, where the light source position is marked by a star.

Several data conversion algorithms map the distribution of ray exit points $(x, y, z)$ as can be seen on a flattened outer skin surface $(x, y)$. This is achieved by generating a central axis in $3 \mathrm{D}$ coordinates along the center of the finger model and converting the cross-sectional coordinates into equivalent angles, thus resulting in a surface plot of position along length in mm. versus position along circumference in degrees (Fig. 2). Surface analysis algorithms split these ray surfaces into square intensity buckets of arbitrary size and collect the total intensity of all rays within each bucket, thus producing an intensity distribution surface (Fig. 2). The static and dynamic intensity surfaces are defined as

$$
\begin{gathered}
I_{(D C)}(x, y)=\sum_{x=x_{a}, y=y_{a}}^{x=x_{b}, y=y_{b}} I_{t(D C)}(x, y), \\
I_{(A C)(\text { sys })}(x, y)=\sum_{x=x_{b}, y=y_{b}, y=y_{a}}^{x} I_{t(A C)(\text { sys })}(x, y), \\
I_{(A C)(\text { dia })}(x, y)=\sum_{x=x_{b}, y=y_{b}}^{x} I_{t(A C)(\text { dia })}(x, y) .
\end{gathered}
$$

Here, $x_{a}=x_{0}-d, y_{a}=y_{0}-d, x_{b}=x_{0}+d$ and $y_{b}=y_{0}+d$, where $\left(x_{0}, y_{0}\right)$ is the position on the flattened finger surface and $d$ is the square bucket half-size. Equations (1-3) yield the ratio of ratios $R$ as a function of surface position:

$$
R(x, y)=\frac{\left(I_{R E D(A C)(\text { dia })}(x, y)-I_{R E D(A C)(s y s)}(x, y)\right) / I_{R E D(D C)}(x, y)}{\left(I_{I R(A C)(\text { dia })}(x, y)-I_{I R(A C)(s y s)}(x, y)\right) / I_{I R(D C)}(x, y)} .
$$

\section{RESULTS}

Simulation results are representative of the in-vivo measurement of a transmission-mode pulse oximeter on a measuring site with an arterial oxygen saturation of $97 \%$. Sensor responses were generated by scanning a square bucket of arbitrary size in $0.1 \mathrm{~mm}$ steps from -1 to $1 \mathrm{~mm}$ longitudinally and from 0 to $360^{\circ}$ circumferentially. All results are averaged over the longitudinal axis.

The SNR in a photoplethysmographic signal is proportional to the peak-to-peak AC intensity. Examination of figure 3 shows that the strongest pulsatile signals can be found at $\pm 60^{\circ}$ to $90^{\circ}$ of the light source position, being approximately 3 times those found at the standard sensor position of transmission pulse oximetry $\left( \pm 180^{\circ}\right)$. 


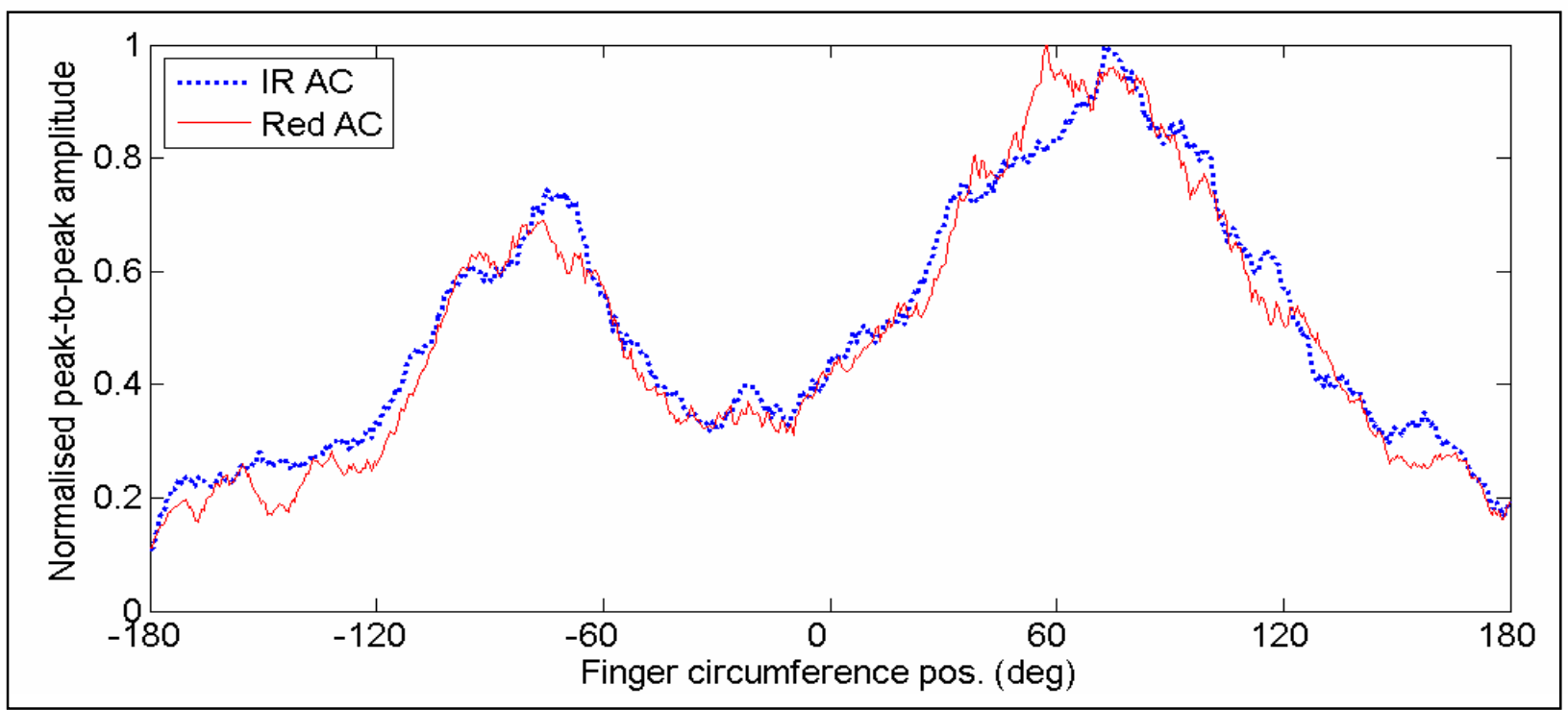

Figure 3. Normalised peak-to-peak AC signals, 2 mm sq. sensor size.

Figure 4 shows the ratio of ratios $R$ generated from both a $2 \mathrm{~mm}$ sq. and a $4 \mathrm{~mm}$ sq. sensor and scaled to $97 \% \mathrm{~S}_{\mathrm{p}} \mathrm{O}_{2}$ $(\mathrm{R}=0.5)$. A scaling factor $S=\alpha / \beta \approx 0.46$ was required to achieve the required saturation. It can be seen that $R$ drops by up to 0.2 , representing a $5 \%$ increase in $\mathrm{S}_{\mathrm{p}} \mathrm{O}_{2}$. This reflects the expected unreliability of oxygen saturation readings between $\pm 45^{\circ}$ of the light source position due to the simulation only taking into account the pulsatility of the main blood vessels. Conversely, saturation readings show stability at $97 \%$ with an error of $\pm 2.5 \%$ for the $2 \mathrm{~mm}$ sensor and of $\pm 1 \%$ for the $4 \mathrm{~mm}$ sensor in the remaining circumference.

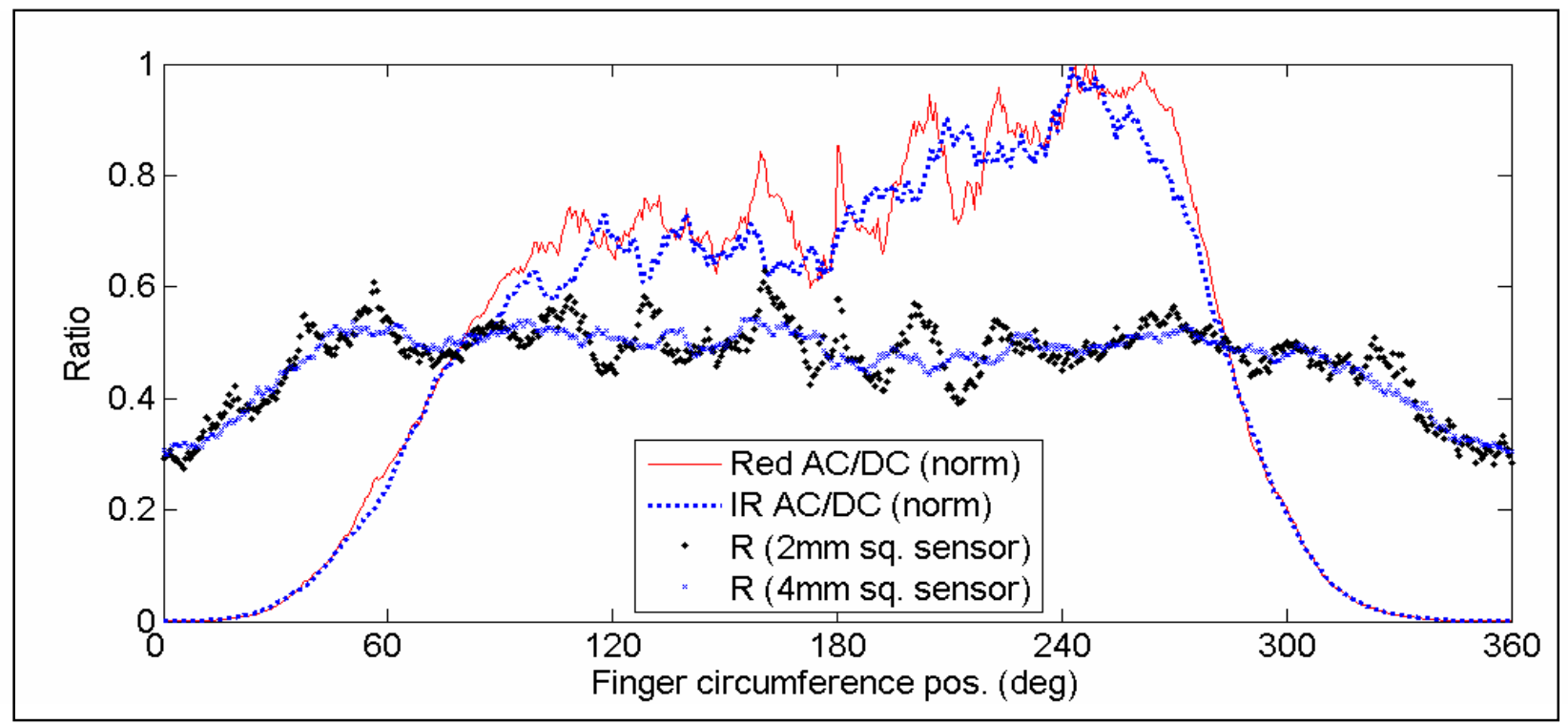

Figure 4. Normalised AC/DC ratios (2mm sq. sensor) and ratio of ratios for data scaled to $97 \% \mathrm{~S}_{\mathrm{p}} \mathrm{O}_{2}(\mathrm{R}=0.5)$

\section{DISCUSSION AND CONCLUSION}

Figure 3 shows a clear asymmetry in the AC responses due to a $1 \mathrm{~mm}-2 \mathrm{~mm}$ offset of the light source with respect to the axis of symmetry of the finger, amounting to a $20 \%$ difference in peak intensity. This is indicative of a potentially significant effect of lateral light-source motion. If quantified, such an effect could be used to characterise motion artefact 
Proceedings of SPIE -- Volume 6446

Biomedical Applications of Light Scattering, Adam Wax, Vadim Backman, Editors, 64460T (Feb. 15, 2007)

in pulse oximetry. The $\mathrm{AC} / \mathrm{DC}$ ratios in figure 4 show the same asymmetry seen in figure 3 . However, the variability of $\mathrm{R}$ can be seen to be inversely proportional to the peak-to-peak AC amplitudes, where greater R instability exists within $\pm 20^{\circ}$ of the standard transmission-mode sensor position and the maximum $\mathrm{R}$ stability coincides with the peaks of figure

3. This indicates optimum sensor positions at $\pm 60^{\circ}$ to $90^{\circ}$ of the light source position in relation to signal quality.

The results of the MC simulation provide insight into the use of multiple sensors in transmittance-mode pulse oximetry for MA characterisation and cancellation. The realistic nature of the results provide support towards the general use of MC simulations in arbitrary geometries to seek useful information into light propagation in optical biomonitoring. However, optimisation of simulation time is essential for the method to be practicable to its full potential.

Empirical validation of the platform presented here is a necessary and forthcoming step in the investigation. Upon its completion, the derivation of equations to characterise changes in light-source and sensor positions as well as changes in optical propertieas and anatomical geometry will be attempted.

\section{ACKNOWLEDGMENTS}

We would like to thank Loughborough University and the Optical Platform (EPSRC 2004-07) for its financial support.

\section{REFERENCES}

1. A. Jubran, "Pulse oximetry", Intensive Care Med. 30(11), 2017-2020 (2004).

2. V. Kamat, "Pulse Oximetry", Indian J. Anaesth. 4 P6U (L4S) E, 2O6X1I-M26E8 (2002).

3. J. Moyle, Principles and Practice Series: Pulse Oximetry, BMJ Publishing Group, London, 1994.

4. P. Mannheimer, J. Casciani, M. Fein, S. Nierlich, "Wavelength selection for low-saturation pulse oximetry", IEEE Trans. Biomed. Eng. 44(3), 148-158 (1997).

5. T. Edrich, M. Flaig, R. Knitza, G. Rall, "Pulse oximetry: an improved in vitro model that reduces blood flowrelated artifacts", IEEE Trans. Biomed. Eng. 47(3), 338-343, (2000).

6. M. Hayes, P. Smith, "A new method for pulse oximetry possessing inherent insensitivity to artefact", IEEE Trans. Biomed. Eng. 48(4), 452-61 (2001).

7. M. Hayes, P. Smith, "Artifact reduction in photoplethysmography”, Appl. Opt. 37(31), 7437-7446 (1998).

8. T. Aoyagi, "Pulse oximetry: its invention, theory, and future", J. Anesth.17, 259-266 (2003).

9. H. Subramanian, B. Ibey, W. Xu, M. Wilson, M. Ericson, G. Cote, "Real-time separation of perfusion and oxygenation signals for an implantable sensor using adaptive filtering”, IEEE Trans. Biomed. Eng. 52(12), 2016$2023(2005)$

10. Y. Mendelson, "Pulse oximetry: theory and applications for noninvasive monitoring", Clin. Chem. 38, 1602-1607 (1992).

11. W. Cheong, S. Prahl, J. Welch, “A Review of the Optical Properties of Biological Tissues”, IEEE J. Quant. Elec. 26(12), 2166-85 (1990).

12. V. Tuchin, Tissue Optics: Light Scattering Methods and Instruments for Medical Diagnosis, SPIE Press, Washington, 2000.

13. A. Lovell, J. Hebden, J. Goldstone, M. Cope, "Determination of the transport scattering coefficient of red blood cells", Proc. SPIE Vol. 3597, Optical Tomography and Spectroscopy of Tissue III. Britton Chance, Robert R. Alfano, and Bruce J. Tromberg, Eds., 175 (1999).

14. A. Bashkatov, E. Genina, V. Kochubey, V. Tuchin, "Optical properties of human skin subcutaneous and mucous tissues in the wavelength range from 400 to $2000 \mathrm{~nm}$ ”, J. Phys. D: Appl. Phys. 38, 2543-2555 (2005).

15. A. Roggan, M. Friebel, K. Dörschel, A. Hahn, G. Müller, "Optical properties of circulating human blood in the wavelength range 400-2500 nm”, J. Biomed. Opt. 4, 36-46 (1999).

16. D. Faber, M. Aalders, E. Mik, B. Hooper, M. van Gemert, T. van Leeuwen, "Oxygen Saturation Dependent Absorption and Scattering of Blood", Phys. Rev. Let. 93(2), 02812 (2004).

17. A. Ugnell, P. Oberg, "The optical properties of the cochlear bone", Med. Eng. Phys. 19, 630-636 (1997).

18. V. Tuchin, S. Utz, I. Yaroslavsky, "Tissue optics, light distribution and spectroscopy", Opt. Eng. 33(10), 31783188 (1994).

19. S. Prahl, http://omlc.ogi.edu/spectra/hemoglobin/index.html

20. I. Sobol, "The distribution of points in a cube and the accurate evaluation of integrals (in Russian)", Zh. Vychisl. Mat. i Mat. Phys. 7, 784-802 (1967). 